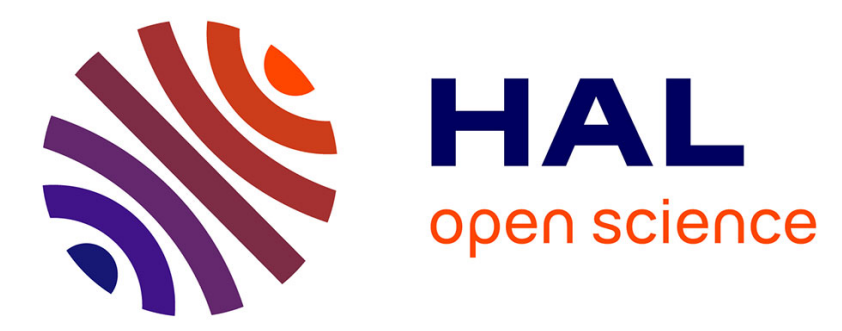

\title{
Les mines-ateliers d'obsidienne de la région de Zinaparo-Prieto, Michoacan, Mexique
}

\author{
V. Darras
}

\section{To cite this version:}

V. Darras. Les mines-ateliers d'obsidienne de la région de Zinaparo-Prieto, Michoacan, Mexique. Bulletin de la Société préhistorique française, 1994, 4-5 (91), pp.290-301. 10.3406/bspf.1994.9738 . halshs-00119711

\section{HAL Id: halshs-00119711 https://shs.hal.science/halshs-00119711}

Submitted on 8 Sep 2020

HAL is a multi-disciplinary open access archive for the deposit and dissemination of scientific research documents, whether they are published or not. The documents may come from teaching and research institutions in France or abroad, or from public or private research centers.
L'archive ouverte pluridisciplinaire HAL, est destinée au dépôt et à la diffusion de documents scientifiques de niveau recherche, publiés ou non, émanant des établissements d'enseignement et de recherche français ou étrangers, des laboratoires publics ou privés. 


\section{Les mines-ateliers d'obsidienne de la région de Zinaparo Prieto,} Michoacan, Mexique

\section{Véronique Darras}

\section{Citer ce document / Cite this document :}

Darras Véronique. Les mines-ateliers d'obsidienne de la région de Zinaparo Prieto, Michoacan, Mexique. In: Bulletin de la Société préhistorique française, tome 91, n4-5, 1994. Le peuplement préhistorique de l'Amérique. pp. 290-301;

doi : https://doi.org/10.3406/bspf.1994.9738

https://www.persee.fr/doc/bspf_0249-7638_1994_num_91_4_9738

Fichier pdf généré le 10/01/2019 


\section{Résumé}

RESUME Localisés au Michoacan, dans le Mexique centre-occidental, les gîtes de la région de Zinaparo-Prieto ont été intensément exploités par les populations préhispaniques. Si l'on constate une utilisation régulière de ces sources d'approvisionnement dès le Précéramique (4000 BP), c'est surtout à la fin du Classique (800 ap. J.-C.) jusqu'au Postclassique moyen (1200 ap. J.-C.) que les stratégies d'exploitation se modifient et que l'on observe une mise en valeur rationnelle et intensive des différents gisements. Le niveau d'organisation des activités d'exploitation implique alors l'existence de groupes bien structurés sur le plan socio-économique tandis que l'échelle de la production, au-delà des nécessités locales, suppose des visées commerciales. Le travail de production se développe dans un espace organisé en différentes aires fonctionnelles : aires d'extraction, ateliers de débitage spécialisés, zones résidentielles. L'approche archéologique de ces complexes d'activités lithiques a requis une méthodologie spécifique adaptée aux conditions particulières du terrain : amoncellements considérables de déchets d'obsidienne, sans formations pédologiques, et s'étendant sur plusieurs hectares. L'analyse des modalités d'exploitation de ces gîtes a indu une reconstitution des chaînes opératoires, depuis l'acquisition de la matière première jusqu'au produit manufacturé.

\section{Resumen}

RESUMEN Localizados al noroeste de Michoacán en el Occidente de Mexico, los yacimientos de obsidiana de la región de Zináparo-Prieto han sido intensamente explotados por las po- blaciones prehispánícas. Aunque su uso se atestigua desde el Precerá- mico (4000 BP), es a partir del Clá- sico Final (800 DC) hasta el Postclá- sico Medio (1200 DC), que se observa una modificación de las estrategias de explotacion, las cuales se traducen por un aprovechamiento intensivo y racional de los yacimientos. Entonces, el grado de organiza- ción de las actividades de explotacion sugiere la existencia de grupos bastante estructurados a nivel socio- económico mientras la escala de producción indica, más alla de las necesidades locales, intenciones commerciales. La produccion se or- ganiza en espacios funcionales bien definidos : areas de mineria, talleres especializados, areas residenciales. El estudio arqueológico de esos centros de producción necesitó una metodología específica (las acumu- laciones de desechos de obsidiana, sin formaciones pedológicas se ex- tienden sobre varias hectareas). El análisis incluyó una reconstitución de las diferentes operaciones desde la adquisición de la materia prima hasta los productos manu-facturados. II peut paraître surprenant d'exposer, dans le cadre d'une journée consacrée au peuplement préhistorique de l'Amérique, des travaux qui concernent une période beaucoup plus récente de l'histoire préhispanique puisqu'elle couvre les quelques siècles qui ont précédé la conquête espagnole. Cependant, la problématique de notre sujet relève largement du champ des études préhistoriques car elle traite du travail de la pierre, en l'occurrence, de mines-ateliers d'obsidienne. Comme la plupart des grands gîtes de Mésoamérique, ceux de la région de Zinaparo-Prieto ont été mis en exploitation de façon rationnelle et intensive par les populations préhispaniques. Leur étude a mis en valeur la complexité des activités de production et le degré de spécialisation révèle des desseins commerciaux et suppose un rôle économique prépondérant au sein des circuits de distribution régionaux et supra-régionaux. Nos recherches s'intègrent dans un vaste programme archéologique commencé en 1983 par le CEMCA (1) au nord-est de l'État du Michoacan. II s'agissait alors d'une contrée peu connue archéologiquement malgré des données historiques rendant compte de son importance culturelle au sein de la Mésoamérique (Miche- let ef alii, 1989). Au moment de la Conquête espagnole, elle faisait partie intégrante du royaume tarasque (ou purepecha) qui s'étendait principalement dans l'état actuel du Michoacan et qui avait assis son pouvoir, au XVe siècle, dans la ville de Tzintzuntzan, à proximité du lac de Patzcuaro (fig. 1). Ce royaume constituait une entité culturelle et politique bien spécifique, qui avait réussi à préserver son autonomie face à la politique expansionniste du pouvoir aztèque.

\section{Abstract}

ABSTRACT Located in the state of Michoacan in Western Mexico, the Zinaparo and Prieto obsidian deposits were intensely exploited by prehispanic populations. The earliest identified use dated from the Preceramic period (4000 BP) but the main exploitation took place from the Late Classic period (800 AD) to the Middle Postclas- sic period (1200 AD). Then, the modifications of economic strategy in the procurement and processing of obsidian were expressed by an intensive and rational exploitation 
which was organized in many functional areas: mining complex, specialized workshops, residential areas. The organisation level in these activities implies the existence of socioeconomical structured groups. The production scale indicates commercial designs beyond the local needs. Archaeological study of these obsidian production centers required adapted methodology (the very big accumulations of obsidian scraps exempt from soil formations spread over several hectareas). The analysis includes a reconstitution of the different operations from the acquisition of raw material to manufactured products. 


\title{
LES MINES-ATELIERS D'OBSIDIENNE DE LA RÉGION DE ZINAPARO-PRIETO, MICHOACAN, MEXIQUE
}

\author{
Véronique DARRAS
}

\section{RÉSUMÉ}

Localisés au Michoacan, dans le Mexique centre-occidental, les gîtes de la région de Zinaparo-Prieto ont été intensément exploités par les populations préhispaniques. Si l'on constate une utilisation régulière de ces sources d'approvisionnement dès le Précéramique (4000 BP), c'est surtout à la fin du Classique (800 ap. J.-C.) jusqu'au Postclassique moyen (1200 ap. J.-C.) que les stratégies d'exploitation se modifient et que l'on observe une mise en valeur rationnelle et intensive des différents gisements. Le niveau d'organisation des activités d'exploitation implique alors l'existence de groupes bien structurés sur le plan socio-économique tandis que l'échelle de la production, au-delà des nécessités locales, suppose des visées commerciales. Le travail de production se développe dans un espace organisé en différentes aires fonctionnelles : aires d'extraction, ateliers de débitage spécialisés, zones résidentielles. L'approche archéologique de ces complexes d'activités lithiques a requis une méthodologie spécifique adaptée aux conditions particulières du terrain : amoncellements considérables de déchets d'obsidienne, sans formations pédologiques, et s'étendant sur plusieurs hectares. L'analyse des modalités d'exploitation de ces gîtes a inclu une reconstitution des chaînes opératoires, depuis l'acquisition de la matière première jusqu'au produit manufacturé.

\section{ABSTRACT}

Located in the state of Michoacan in Western Mexico, the Zinaparo and Prieto obsidian deposits were intensely exploited by prehispanic populations. The earliest identified use dated from the Preceramic period (4000 BP) but the main exploitation took place from the Late Classic period (800 AD) to the Middle Postclassic period (1200 AD). Then, the modifications of economic strategy in the procurement and processing of obsidian were expressed by an intensive and rational exploitation which was organized in many functional areas: mining complex, specialized workshops, residential areas. The organisation level in these activities implies the existence of socioeconomical structured groups. The production scale indicates commercial designs beyond the local needs. Archaeological study of these obsidian production centers required adapted methodology (the very big accumulations of obsidian scraps exempt from soil formations spread over several hectareas). The analysis includes a reconstitution of the different operations from the acquisition of raw material to manufactured products.

\section{RESUMEN}

Localizados al noroeste de Michoacán en el Occidente de Mexico, los yacimientos de obsidiana de la región de Zináparo-Prieto han sido intensamente explotados por las poblaciones prehispánicas. Aunque su uso se atestigua desde el Precerámico (4000 BP), es a partir del Clásico Final (800 DC) hasta el Postclásico Medio (1200 DC), que se observa una modificación de las estrategias de explotacion, las cuales se traducen por un aprovechamiento intensivo y racional de los yacimientos. Entonces, el grado de organización de las actividades de explotacion sugiere la existencia de grupos bastante estructurados a nivel socioeconómico mientras la escala de producción indica, más allá de las necesidades locales, intenciones commerciales. La produccion se organiza en espacios funcionales bien definidos : areas de minería, talleres especializados, areas residenciales. El estudio arqueológico de esos centros de producción necesitó una metodología específica (las acumulaciones de desechos de obsidiana, sin formaciones pedológicas se extienden sobre varias hectareas). El análisis incluyó una reconstitución de las diferentes operaciones desde la adquisición de la materia prima hasta los productos manufacturados.
II peut paraître surprenant d'exposer, dans le cadre d'une journée consacrée au peuplement préhistorique de l'Amérique, des travaux qui concernent une période beaucoup plus récente de l'histoire préhispanique puisqu'elle couvre les quelques siècles qui ont précédé la conquête espagnole. Cependant, la problématique de notre sujet relève largement du champ des études préhistoriques car elle traite du travail de la pierre, en l'occurrence, de mines-ateliers d'obsidienne. Comme la plupart des grands gîtes de Mésoamérique, ceux de la région de Zinaparo-Prieto ont été mis en exploitation de façon rationnelle et intensive par les populations préhispaniques. Leur étude a mis en valeur la complexité des activités de production et le degré de spécialisation révèle des desseins commerciaux et suppose un rôle économique prépondérant au sein des circuits de distribution régionaux et supra-régionaux.

Nos recherches s'intègrent dans un vaste programme archéologique commencé en 1983 par le CEMCA (1) au nord-est de l'État du Michoacan. II s'agissait alors d'une contrée peu connue archéologiquement malgré des données historiques rendant compte de son importance culturelle au sein de la Mésoamérique (Michelet et alii, 1989). Au moment de la Conquête espagnole, elle faisait partie intégrante du royaume tarasque (ou purepecha) qui s'étendait principalement dans l'état actuel du Michoacan et qui avait assis son pouvoir, au $X V^{e}$ siècle, dans la ville de Tzintzuntzan, à proximité du lac de Patzcuaro (fig. 1). Ce royaume constituait une entité culturelle et politique bien spécifique, qui avait réussi à préserver son autonomie face à la politique expansionniste du pouvoir aztèque.

(1) La problématique initiale de l'équipe du CEMCA (Centre d'Études Mexicaines et Centraméricaines) était la réalisation d'une étude régionale visant à reconstituer les modalités de l'occupation humaine tout en définissant son cadre spatio-temporel, afin de la replacer dans le contexte mésoaméricain. 


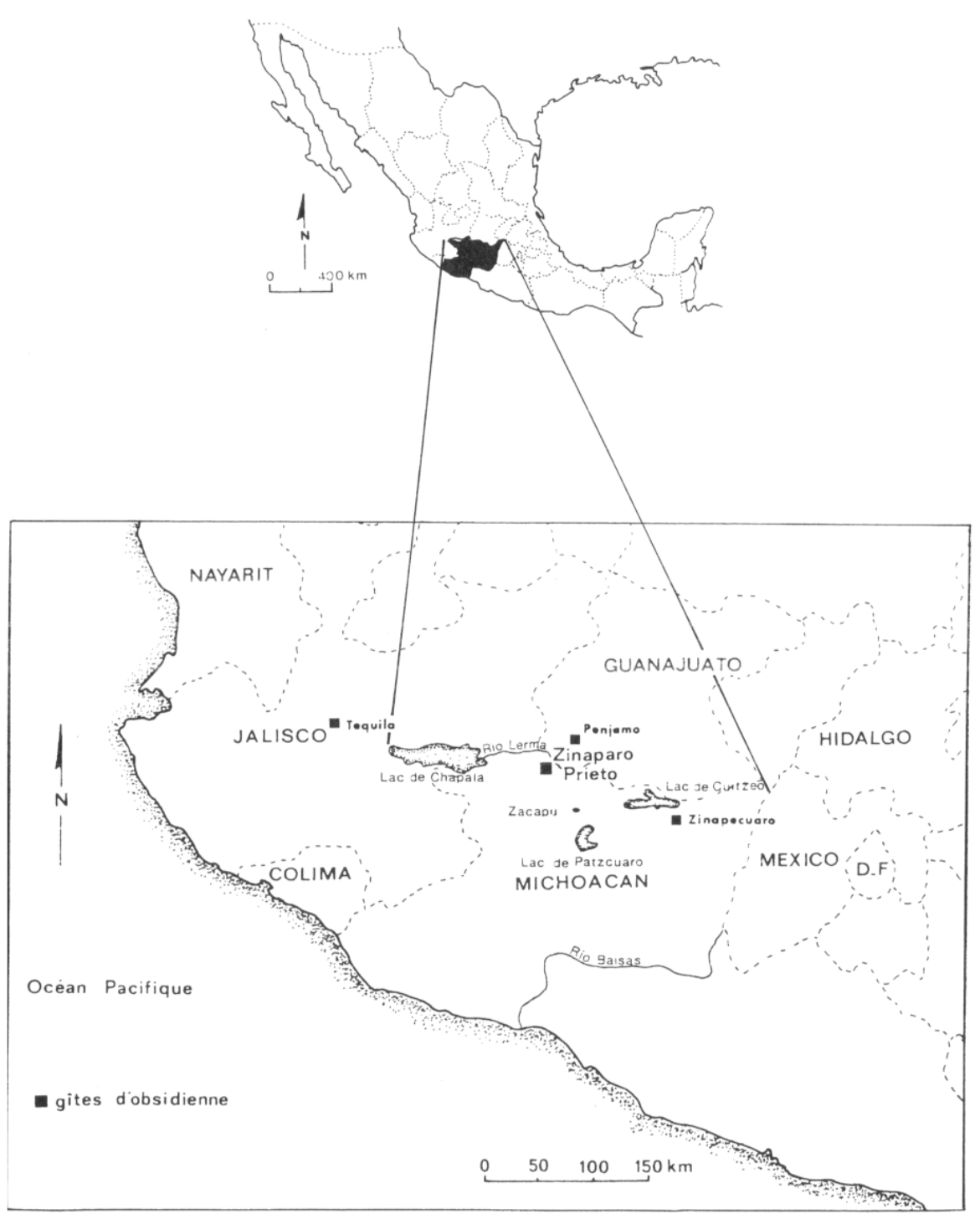

Fig. 1 - Localisation de la région d'études dans le Centre-Nord du Michoacan.

volcan ayant été recouverte par les laves andésitiques du Cerro Grande. Les formes de gisements sont diverses : affleurements de veines massives qui peuvent atteindre 4 mètres d'épaisseur, concentrations de nodules roulés libérés par les processus d'érosion, nodules prisonniers de conglomérats rhyolitiques ou de gangues friables et vacuolaires, à forte teneur en silice. II s'agit d'obsidiennes de bonne qualité pour la taille, avec peu d'inclusions internes, de teintes prédominantes rouge-brun et/ou noires et grises.

Au sud du massif, les magmas du Cerro Varal, également recouverts par les épanchements andésitiques du Cerro Grande, ont généré des obsidiennes consolidées en veines massives et homogènes, ou bien se présentant sous l'aspect de blocs pris dans une matrice rhyolitique. II s'agit d'obsidiennes très pures, d'un gris translucide ou opaque avec parfois des veinures plus foncées.

Les obsidiennes du Cerro Prieto, dôme rhyolitique dont le sommet est totalement recouvert par un appareil andésitique, s'épanchent dans la partie aval de la face nord. II s'agit le plus souvent de gisements de surface présentant des concentrations importantes de nodules libérés par les processus érosifs. L'obsidienne est partiellement cristallisée, de couleur gris-bleuté, rêche au toucher et d'aspect mat.

\section{Les résultats de la prospection}

\section{PRÉSENTATION GÉNÉRALE}

\section{- Le cadre géographique et géologique}

Le massif de Zinaparo-Churintzio et le volcan du Cerro Prieto sont localisés au centre-nord du Michoacan, à $25 \mathrm{~km}$ au sud de la ville de La Piedad, entre $101^{\circ} 59^{\prime}$ et $102^{\circ} 04^{\prime}$ de longitude Ouest et entre $20^{\circ} 02^{\prime}$ et $20^{\circ} 10^{\prime}$ de latitude Nord (fig. 1). Situé dans la partie méridionale du Bajio montagneux et intégré dans la dépression du rio Lerma, cet ensemble montagneux appartient géologiquement à l'axe néovolcanique transmexicain, constitué progressivement durant le Quaternaire (Demant, 1981, 1983).

La sous-région étudiée couvre environ $100 \mathrm{~km}^{2}$ et comporte plusieurs formations volcaniques de chronologie et de nature distinctes, d'altitude variant entre $1800 \mathrm{~m}$ et $2600 \mathrm{~m}$. On y observe des formations basiques de type andésitique et surtout un volcanisme acide, plus ancien, et qui a produit successivement une série d'appareils de nature rhyolitique.

L'étude géologique et pétrographique, complétée par la caractérisation géochimique d'échantillons d'obsidienne prélevés in situ dans les affleurements, a abouti à la distinction de trois dômes rhyolitiques ayant généré chacun des obsidiennes de composition spécifique (Demant, 1989, Joron et alii, 1990).

Les obsidiennes formées lors des émissions acides du Cerro Zinaparo occupent essentiellement le front des coulées, sur les flancs nord et nord-est du massif, la partie sud du
Grâce aux indications fournies par l'étymologie du terme Zinaparo qui désigne le "lieu de l'obsidienne" en langue purepecha (de tzinapu : obsidienne, suffixe locatif ro : lieu), la localisation de ces gîtes fut grandement facilitée lors des premières explorations effectuées dans la région. Ce village avait déjà été signalé à deux reprises comme source potentielle de matière première sans toutefois avoir été visité (Ericson et Kimberlin, 1977 ; Pollard Perlstein, 1982). La reconnaissance méthodique qui a suivi cette approche préliminaire du massif de Zinaparo et de ses abords, s'est déroulée avec le concours d'informateurs locaux et a permis le recensement de 44 sites archéologiques, dont 16 centres d'exploitation de l'obsidienne concentrés dans les parties nord-est, est et sud du massif, ainsi que sur 


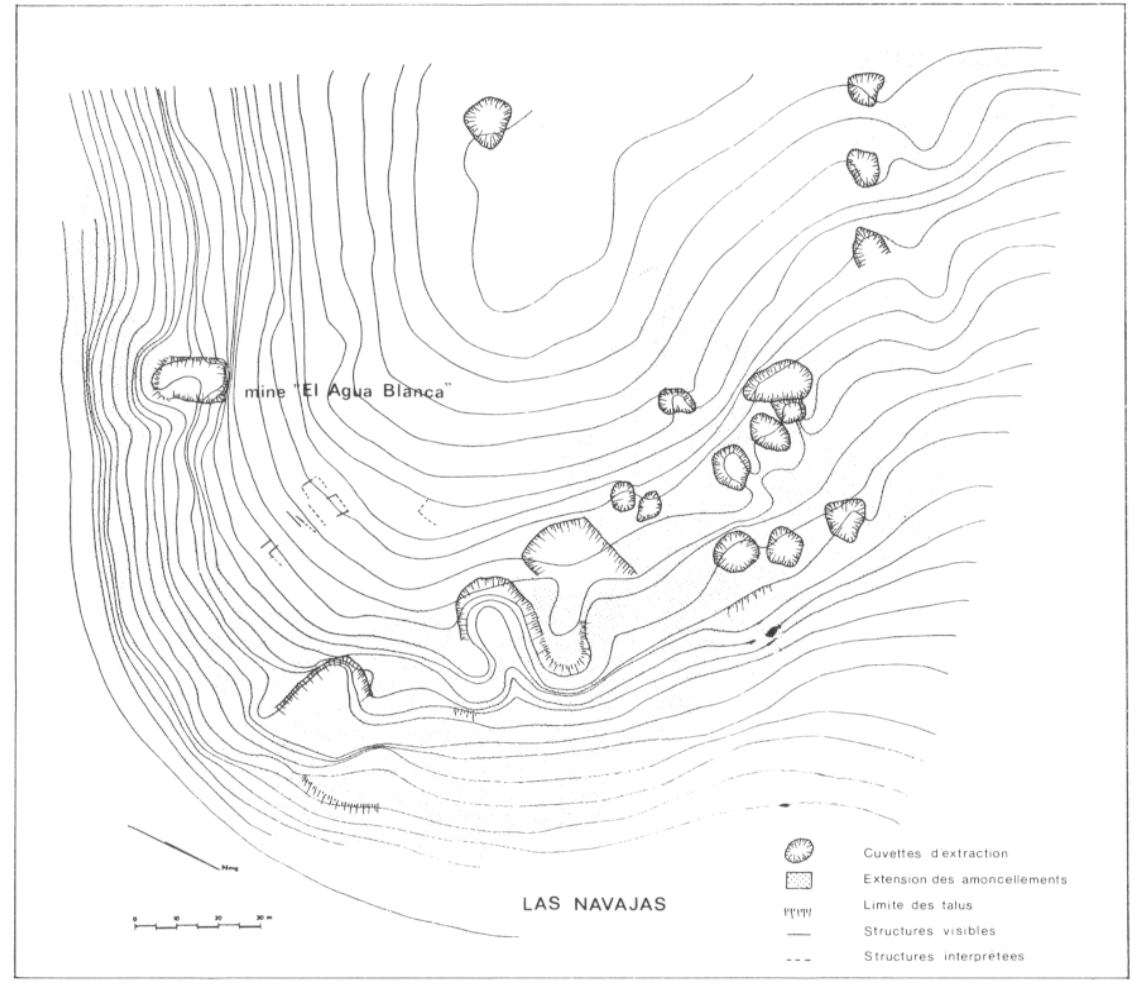

Fig. 2 - Plan topographique du centre d'exploitation de "Las Navajas".

les flancs nord du Cerro Prieto (2). Chaque centre identifié constitue une entité de production organisée, qui inclut des espaces fonctionnels bien définis : aires de récoltes des blocs, aires d'extraction, ateliers de débitage, aires de rejet des déblais et parfois aires d'habitats (fig. 2). Les autres sites répertoriés sont de natures diverses et occupent plus volontiers les bordures sud-ouest, ouest et nord du massif : on y a identifié des établissements avec des restes d'architecture (3) offrant en surface un matériel archéologique plus ou moins dense (matériel céramique et lithique), quelques abris sous roche et grottes présentant des vestiges d'occupation, des sites définis par des concentrations en surface de matériel archéologique (également de la céramique et du lithique), des ensembles de pétroglyphes (Darras, 1989 ; Darras et Monnet, 1990) ainsi qu'une car-

(2) Huit centres d'exploitation sont rattachés au Cerro Zinaparo, sept s'étendent sur les flancs du Cerro Varal et une zone de grande extension comprenant plusieurs concentrations a tété identifiée sur le Cerro Prieto.

(3) On y rencontre principalement des plates-formes construites qui soutiennent des restes de maisons, mais aussi des structures dites cérémonielles (jeux de balles, monticules, etc.) et des terrasses agricoles. rière de pierre exploitée pour la construction.

Le bilan consécutif à une classification plus précise de ces entités archéologiques met donc en valeur deux secteurs principaux, bien délimités géographiquement et à vocation distincte : d'une part, un secteur comprenant des sites spécialisés, consacrés à une activité économique quasi-exclusive, l'exploitation de l'obsidienne. D'autre part, un secteur principalement occupé par des hameaux habités par des populations sédentaires, peut-être liées au travail de l'obsidienne mais assurant leur subsistance quotidienne grâce aux activités agricoles.

\section{Objectifs et méthodologie}

Dans une première étape, les travaux qui ont porté sur les gîtes d'obsidienne avaient trois objectifs : l'approche géologique de la région étudiée suivie de l'échantillonnage des sources afin de procéder aux caractérisations physico-chimiques nécessaires, le placement temporel du gros de l'exploitation et de l'occupation humaine dans le massif, et enfin, l'étude des modalités d'exploitation des gîtes. Celle-ci visait à comprendre le fonctionnement des centres d'exploitation et à reconstituer globalement les différentes étapes de la production, depuis l'acquisition de la matière première jusqu'au produit manufacturé prêt à être écoulé. En accord avec la perspective régionale du programme, il s'agissait avant tout de rendre compte d'un phénomène d'ensemble, de saisir les grandes articulations fonctionnelles de ces secteurs d'activités et d'en inférer les interactions socio-économiques. Cette étude devait permettre d'établir les bases de recherches ultérieures appelées à explorer de façon plus circonstanciée certains aspects, ou bien à envisager les épisodes consécutifs à la production et propres à la commercialisation.

L'approche archéologique de tels complexes d'activités a requis une méthodologie adaptée aux conditions du terrain et a impliqué certains choix en accord avec la problématique de départ. Les vestiges miniers et les amoncellements considérables de déchets de taille, qui témoignent de l'intensité des activités d'exploitation, s'intègrent dans un plan d'ensemble cohérent et modifient fortement la topographie originelle du terrain en marquant de façon notable l'ensemble du paysage (4) (fig. 3). A titre d'exemple, la plus petite concentration (La Mata de Casirpes) s'étend sur près de $500 \mathrm{~m}^{2}$ tandis que l'extension globale des Tzinapus del Cerro Prieto atteint presque 8 ha. Les accumulations de déchets dérivés de l'extraction et du débitage qui peuvent atteindre un à deux mètres d'épaisseur, voire plus sur certaines buttes, n'incluent pas ou peu de formations pédologiques (lorsqu'elles existent, ces formations sont en général superficielles). Comment aborder alors de façon cohérente ces sites de grande extension qui se traduisent par des milliers de tonnes de déchets d'obsidienne? Dans un tel contexte, ces particularités excluaient bien évidemment une approche traditionnelle relevant des méthodes de fouilles préhistoriques. Par ailleurs, les études de surface ne pouvaient, à elles seules, rendre compte de certains phénomènes : profondeur des dépôts d'obsidienne, variations qualitatives et quantitatives au sein des amoncellements.

(4) En maintenant un certain taux d'humidité, les amoncellements d'obsidienne ont favorisé la croissance et la généralisation d'une espèce de fougères qui est, pour l'archéologue, un indicateur infaillible. 


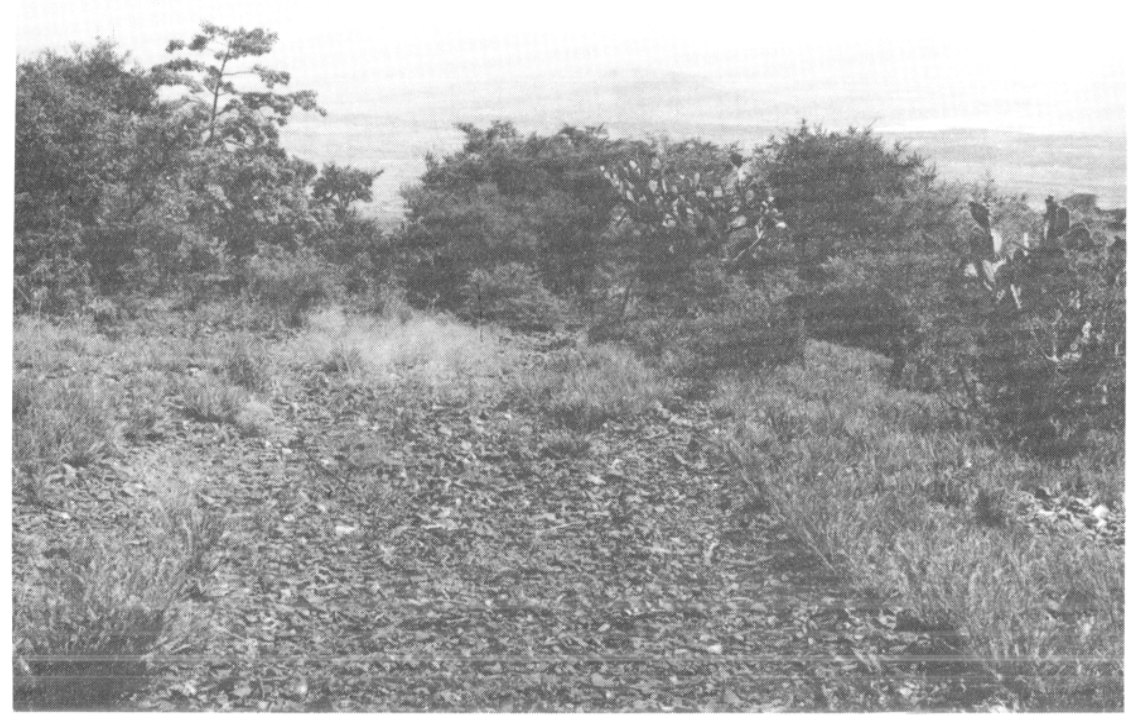

Fig. 3 - Vue des ateliers de débitage de "Las Cocinas".

Parmi les différentes alternatives, comportant chacune des limites, les démarches suivantes ont été adoptées :

* des relevés topographiques ont été effectués dans les sites les plus importants, afin de mettre en valeur les divers ensembles fonctionnels et leurs interactions, ainsi que d'éventuelles concentrations différentielles ;

* des parcours méthodiques ont permis une couverture complète de toutes les zones d'exploitation, accompagnés des descriptions nécessaires et de ramassages sélectifs ;

* des sondages stratigraphiques ont été réalisés dans des aires d'extraction et dans des ateliers de débitage (sur les talus et dans les pentes), sélectionnés en fonction de leur nature et importance, afin de connaître l'épaisseur et la nature des dépôts d'obsidienne, d'en vérifier l'homogénéité ou la diversité (surface/profondeur) et d'obtenir un échantillon représentatif du matériel pour l'analyse techno-morphologique. L'extrême abondance du matériel a incité à faire plusieurs types de ramassage : systématiques ou sélectifs ; en outre, dans plusieurs cas, une classification a été effectuée sur place, suivie d'un échantillonnage représentatif (5).

* des sondages stratigraphiques ont été effectués dans les sites d'ha-

(5) A titre d'exemple, la comptabilisation systématique du matériel sorti de l'un des sondages a donné un total de 24601 pièces, pour un volume fouillé de $1,50 \times 1,50 \times 0,50 \mathrm{~m}$. bitat (associés aux zones d'exploitation ou non).

\section{L'ACQUISITION DES BLOCS}

Plusieurs modes d'obtention de la matière première ont été identifiés, le plus souvent au sein d'un même centre de production. Le contexte géologique a permis à la fois une exploitation en mines souterraines et à ciel ouvert. Conjointement à ces activités minières, on observe des aires assez vastes et bien délimitées où se concentrent en surface des quantités importantes de nodules roulés, libérés par les processus érosifs. Ces nodules, de taille plutôt réduite (entre $500 \mathrm{gr}$ et $2 \mathrm{~kg}$ ) et au cortex raviné, ont été également ramassés puis exploités dans les ateliers de débitage.

\section{Les exploitations souterraines}

Six localités présentent des vestiges d'extraction souterraine, mais seules deux mines sont restées entièrement accessibles, les autres ayant été obstruées par des éboulements de blocs rhyolitiques. Toutes ces unités d'extraction ont été creusées dans des veines massives, le plus souvent homogènes, et qui renferment des blocs d'obsidienne de grande taille. Le désir d'acquérir ces gros blocs, peu altérés et d'excellente qualité pour la taille, a été déci- sif dans le choix du mode d'exploitation. Par ailleurs, les coloris ont pu être un critère déterminant : l'obsidienne extraite de ces exploitations est rouge-brun, noire ou gris translucide. Suivant les conditions topographiques et la position de la coulée d'obsidienne, ces mines requéraient parfois de gros travaux préliminaires qui visaient à atteindre et dégager la veine, puis à aménager un espace ouvert et plat destiné à faciliter les activités minières : évacuation des déchets dérivés de l'extraction, transport des blocs, circulation des mineurs.

En profitant le plus souvent des fractures naturelles dans les épanchements rhyolitiques ou d'obsidienne, les exploitants effectuaient deux coupes verticales, ajustées perpendiculairement, jusqu'à la mise au jour de la veine ou de sa partie exploitable. Ces parois, hautes de 3 à $4 \mathrm{~m}$, contribuaient à l'agencement de l'espace protégé qui allait abriter les activités d'extraction. Dans le même but, l'aplanissement du sol attenant facilitait ensuite la mise en place d'une zone de circulation devant l'entrée de la future mine. Une fois ces préparations effectuées, l'ouverture de la mine était assurée et des solutions techniques étaient apportées aux problèmes que posaient son extension progressive (mise en place de colonnes de soutènement, entrées secondaires favorisant la ventilation et la circulation). Au fur et à mesure de l'extraction, une partie des déchets (gangue, fragments non exploitables, petits blocs, éclats) était laissée sur place tandis que le reste était rejeté hors de la mine, dans la pente située à l'extrémité de la zone de circulation, créant ainsi progressivement un talus de déjection. Peu d'indices concernant les modes d'extraction ont pu être observés : le percuteur aménagé sur dalle d'andésite, recueilli dans l'une des mines et ceux rencontrés à proximité des aires d'extraction faisaient sans doute partie de l'équipement du mineur. Selon la nature des coulées (veines homogènes ou blocs prisonniers d'une matrice), les mineurs ont pu aussi dégager les blocs à l'aide de leviers et de pics de bois (Cobean et Stocker, 1984 ; Pastrana, 1987) (6). Une fois libérés, ces blocs

(6) A l'intérieur des mines d'obsidienne du Pico de Orizaba (Etat de Veracruz, Mexique) des pics en bois de pin ont été retrouvés. L'un d'eux mesurait $2 \mathrm{~m}$ de long pour $15 \mathrm{~cm}$ de section. Plusieurs percuteurs de pierre y ont également été recueillis. 
étaient souvent dégrossis sur place, leur allègement facilitant leur transport hors de la mine.

Les deux mines bien accessibles présentent les caractéristiques suivantes:

- La mine de La Guanumeña, est située au sein d'une coulée massive qui affleure dans une pente fort inclinée. Creusée dans l'une des parois d'attaque, elle comporte deux entrées adjacentes, l'une donnant sur l'étendue aménagée et l'autre s'ouvrant sur le versant. L'ouverture principale $(2 \times 1,20 \mathrm{~m})$ donne accès à une antichambre d'environ $20 \mathrm{~m}^{2}$ qui se prolonge par une galerie longue de $24 \mathrm{~m}$ et haute en moyenne de $1 \mathrm{~m}$ par rapport au sol de remplissage. Celui-ci est composé des déchets produits lors de l'extraction : sables d'obsidienne, morceaux de gangue, débris naturels, petits nodules. D'après nos tentatives d'évaluations quantitatives, le volume global creusé dans cette mine a été d'au moins $260 \mathrm{~m}^{3}$ et 605 tonnes de matériaux ont dû en être extraits, dont 237 tonnes d'obsidienne utile (Darras, 1991). Les nodules, (majoritairement de couleur rouge-brun et/ou noire), sont prisonniers d'une gangue friable, facilitant l'extraction. Les nodules extraits et sélectionnés pesaient entre 2 et $15 \mathrm{~kg}$, les plus petits étant abandonnés sur place.

- La mine de El Agua Blanca a été creusée au travers d'une veine massive dégagée dans une coulée rhyolitique, à $4 \mathrm{~m}$ de profondeur. Elle est constituée de deux chambres principales et de plusieurs boyaux. L'extraction s'orientait en effet au gré de la qualité apparente des blocs dans les parois et elle était guidée par l'existence de fractures naturelles qui facilitait leur dégagement. La surface au niveau du sol de remplissage est d'environ $120 \mathrm{~m}^{2}$, les hauteurs variant entre $0,50 \mathrm{~m}$ et $1,70 \mathrm{~m}$. Le volume global creusé s'élève à près de $400 \mathrm{~m}^{3}$ et plus de 900 tonnes de matériaux en ont été extraits, la moitié correspondant aux déchets constituant le sol de remplissage et le talus de déjection, l'autre consistant en la masse d'obsidienne utile. La veine d'obsidienne, amplement fracturée après sa consolidation, renferme des blocs angulaires pouvant atteindre un poids de $25 \mathrm{~kg}$.

\section{- Les exploitations à ciel ouvert}

Ce type d'extraction est le plus répandu dans les gîtes de la région de
Zinaparo-Prieto. II consiste en l'excavation ponctuelle d'affleurements, le plus souvent jusqu'à épuisement de l'épaisseur de la veine. D'après les observations de surface, il s'agissait de gisements massifs ou de blocs apparaissant dans des conglomérats rhyolitiques. Les vestiges $\mathrm{mi}-$ niers qui en résultent sont des cuvettes ou des tranchées plus ou moins profondes, de taille et de morphologie variables. Les cuvettes d'extraction se répartissent sur les versants, face à la pente, au milieu des ateliers de débitage. Leur nombre varie en fonction de l'extension et de la qualité des affleurements : une seule dans le site de la Mata de Casirpes, dix dans le site de Las Cocinas et 35 recensées dans le site des Tzinapus del Cerro Prieto. II est difficile de se représenter l'aspect originel de ces unités d'extraction car elles ont été comblées par des éboulements d'obsidienne provenant des ateliers contigüs. D'après leur configuration actuelle, ces cuvettes ont été classées en trois types morphologiques : les cuvettes de petite taille, de diamètre compris entre 4 et $10 \mathrm{~m}$ et de profondeur n'excédant pas $1 \mathrm{~m}$, les cuvettes de même morphologie mais de longueur comprise entre 10 et $15 \mathrm{~m}$ et de profondeur variant entre $1 \mathrm{~m}$ et $1,50 \mathrm{~m}$ et enfin les dépressions profondes (entre 1 et $2 \mathrm{~m}$ ), dépassant les $15 \mathrm{~m}$ de longueur et fermées dans leur extrémité aval par des buttes.

\section{LES ATELIERS DE DÉBITAGE}

Les unités d'extraction souterraine peuvent se situer à proximité immédiate des aires de débitage, qui occupent alors les terrains attenants mais peuvent également être plus distantes, ce qui supposait dans ce cas, l'acheminement des blocs jusqu'au lieu de leur transformation. Au nord-est du massif, la zone d'exploitation de La Guanumeña assurait essentiellement les activités d'extraction et de dégrossissage des blocs même si, ponctuellement, des activités de débitage se sont déroulées dans l'entrée et à la sortie de la mine (notamment pour la fabrication de préformes bifaciales). L'absence d'ateliers de débitage à proximité incite à penser que les blocs étaient transportés vers d'autres lieux plus éloignés, hors du massif de ZinaparoChurintzio. De même, la mine de $E l$
Agua Blanca, intégrée dans une zone d'exploitation très étendue (Las $\mathrm{Na}$ vajas ; fig. 2), se situe à une trentaine de mètres des premiers ateliers, la forte pente à proximité de la mine ayant empêché toute installation pour les activités de débitage.

En revanche, à chaque unité d'extraction à ciel ouvert correspond une aire de débitage, installée sur les pourtours et s'étendant sur les pentes douces attenantes. La multiplication de tels ensembles a engendré des épandages uniformes, avec peu d'interruptions spatiales. L'imbrication de ces deux unités fonctionnelles (mines/ateliers) impliquait donc un minimum de circulation et probablement un enchaînement des actions. L'extraction était aussitôt suivie du débitage : les artisans récupéraient les blocs fraîchement extraits, parfois déjà entamés (enlèvements provoqués lors de l'extraction ou test de qualité ?) et assuraient instantanément un dégrossissage suivi des autres étapes du débitage. Des blocs pouvaient être stockés sur le lieu du débitage dans l'attente de leur exploitation. Le déblayage des unités d'extraction s'effectuait conjointement aux autres tâches, les déchets provenant de ces cuvettes étant souvent évacués au sein même des ateliers ou dans les aires de rejet. Toutes ces opérations fonctionnaient donc en symbiose et il n'est pas impossible qu'elles aient été assurées alternativement par les mêmes individus, sans répartition spécifique des tâches (extraction, déblayage, débitage). Seul le travail en mines, beaucoup plus complexe et long, a dû faire appel aux savoir-faire de spécialistes. L'extrémité aval des plus grandes dépressions d'extraction à ciel ouvert constituait un lieu d'installation priviligié et les accumulations sur place des déchets de débitage ont formé des petites proéminences qui surplombent d'imposants talus de déjection (fig. 4). Les artisans s'appliquaient à y rejeter les plus gros déchets (la pesanteur favorisant de toute façon un charriage naturel dans le bas de ces pentes), de façon à désencombrer l'espace de débitage, qui pouvait servir de zone de passage, mais aussi à éviter des éboulements dans l'unité d'extraction.

A l'heure actuelle, on observe donc une distribution différentielle du matériel qui résulte de l'attribution fonctionnelle des différents espaces mais aussi de phénomènes naturels : 


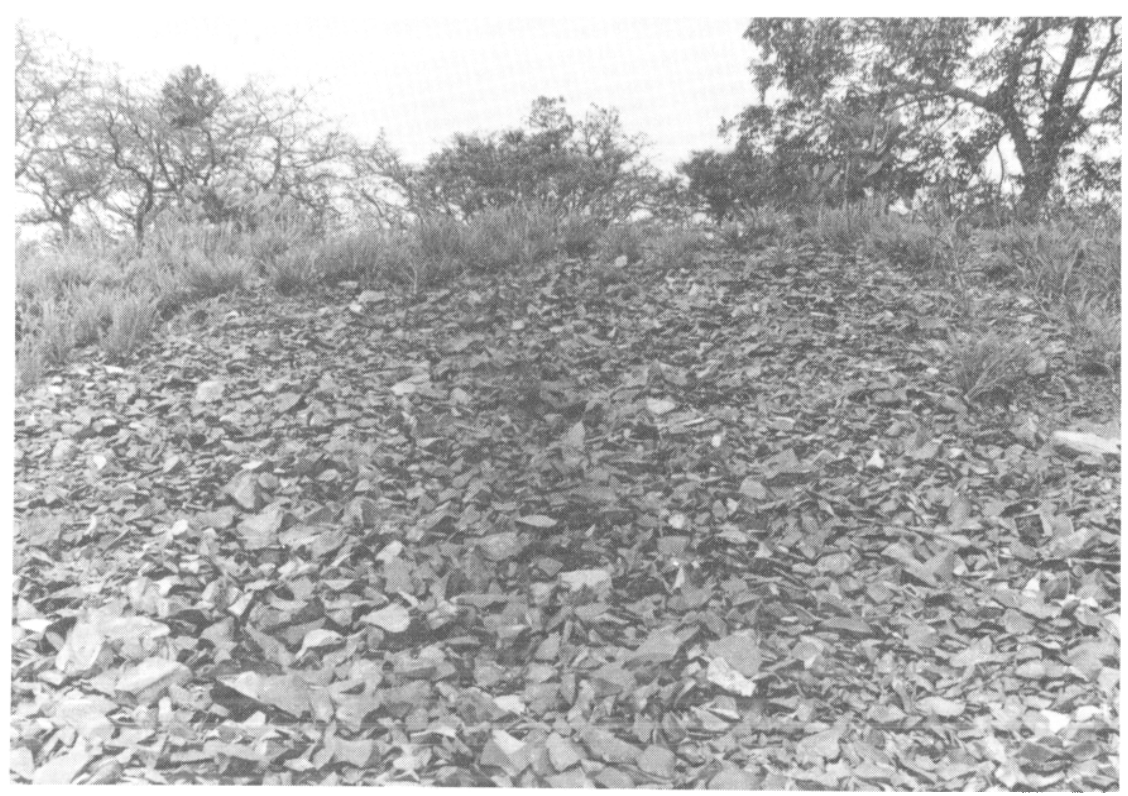

Fig. 4 - Talus de déjection dans les ateliers des "Tzinapus del Cerro Prieto".

les plus gros déchets se concentrent en périphérie des zones d'exploitation, dans le bas des pentes et dans les talus de déjection. Les remblais qui composent actuellement les cuvettes d'extraction renferment majoritairement des gros déchets provenant des éboulis des ateliers contigüs. Par contre, de tels mouvements sont moins perceptibles dans les épandages qui occupent des terrains plus nivelés.

Finalement, l'essentiel de ces zones de débitage renferme de $40 \%$ à $90 \%$ de déchets taillés et de résidus produits simultanément au débitage (esquilles et poussières d'obsidiennes). Le reste est composé de la matière première brute ou dégrossie destinée à servir de support au débitage, des débris inexploitables (petits nodules, débris naturels d'obsidienne, fragments de rhyolites), de la terre et des débris végétaux (toujours moins de $20 \%$ ) et enfin, certains des outils des artisans, en l'occurrence des percuteurs d'andésite. Au sein de la masse de déchets taillés, on identifie surtout les éclats qui résultent des activités de préparation et de réfection. Les produits qui constituent la finalité du débitage sont censés être absents puisqu'évacués hors des ateliers. Toutefois, lorsqu'on les retrouve, ils peuvent être considérés comme des rebuts, délaissés le plus souvent pour des raisons précises : if s'agit de produits imparfaits (ne correspondant pas aux normes requises) ou inutilisables (accidents de taille, irrégularités, fractures consécutives au détachement etc.).
La grande uniformité apparente de ces milliers de tonnes de déchets semble correspondre à une production artisanale réalisée avec constance par des spécialistes, qui devaient se consacrer essentiellement à cette tâche. Toutefois, cette spécialisation d'ensemble peut occulter des formes de production secondaires et plus diversifiées, réalisées ou non par les mêmes groupes de gens, à la même époque ou non.

Toujours est-il que les observations de surface indiquent que les ateliers de 14 centres d'exploitation fabriquaient globalement le même type de produits avec des méthodes de débitage similaires (d'après la terminologie définie par J.Tixier et alii, 1980). Ces ateliers sont tous localisés dans le massif de Zinaparo et se rattachent aux deux gîtes principaux du Cerro Zinaparo et du Cerro Varal. Par contre, les ateliers installés sur les pentes du Cerro Prieto avaient une production distincte qui résulte probablement des contraintes relatives aux caractéristiques de son obsidienne. Confortant ces constatations, les sondages réalisés dans les ateliers ont permis de vérifier l'homogénéité de ces dépôts d'obsidienne, de la surface jusqu'au substratum, des fluctuations au sein des classes d'objets intervenant uniquement sur le plan quantitatif.

Suite à ces remarques préliminaires, l'analyse plus détaillée des activités de débitage permettant la reconstitution théorique des grandes étapes de fabrication, a porté sur l'analyse techno-morphologique des produits recueillis dans les sondages de 4 sites : il s'agit de Las Cocinas, Las Navajas, La Mata de Casirpes, tous les trois installés sur les pentes du Cerro Varal et du site des Tzinapus del Cerro Prieto.

\section{Les ateliers du Cerro Varal}

Les observations relatives à la nature des produits constituant les amoncellements des ateliers du Cerro Varal permettent de constater :

- la grande proportion d'éclats de débitage aux morphologies très diversifiées (de $48 \%$ à $59 \%$ des déchets) ;

- l'importance des produits laminaires (de $32 \%$ à près de $50 \%$ ), qui présentent également une grande variation dans leurs caractéristiques morphologiques ; on y constate la faible représentativité de lames régulières ;

- le très grand nombre de nucléus à lames résiduels de configuration finale "plate" (fig. 5a) ; en surface, on peut comptabiliser jusqu'à une vingtaine de nucléus sur une surface de $20 \mathrm{~m}^{2}$. A titre d'exemple, le sondage $\mathrm{C}$ dans le site de Las Cocinas (environ $1 \mathrm{~m}^{3}$ fouillé) en a fourni 22 exemplaires ;

- la présence de petits nucléus coniques se trouvant à l'état résiduel et ayant servi de support au débitage de petites lames (4 exemplaires dans le même sondage) ;

- la présence de nucléus coniques configurés et prêts à l'exploitation laminaire (fig. 5 b) ;

- la présence d'outils unifaciaux sur grands supports laminaires mais, bien entendu, leur fréquence est faible par rapport à la masse des déchets (fig. 5 e) ;

- la faible fréquence de préformes bifaciales, mais l'existence de déchets attestant la pratique d'un façonnage bifacial ;

- la présence exceptionnelle d'outils sur éclats.

Ces examens de surface, renforcés par l'analyse du matériel recueilli dans les sondages, ont permis d'inférer que la finalité du débitage consistait en la fabrication, par per- 


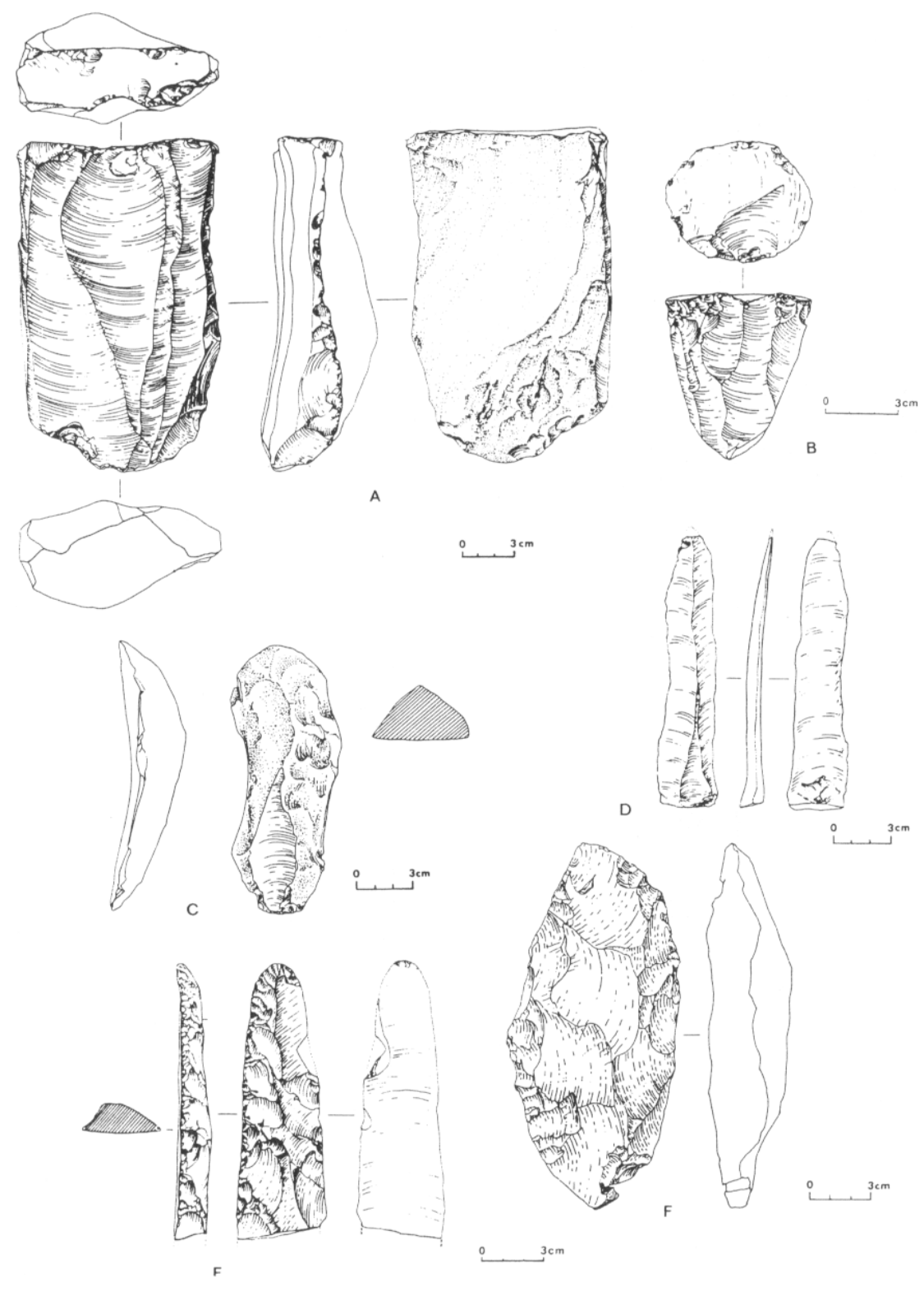

Fig. 5 - (a) nucléus plats ; (b) nucléus coniques ; (c) produit laminaire cortical ; (d) lame brute ; (e) outil unifacial sur lame; (f) préforme bifaciale.

cussion (7), de supports laminaires aux modules variables, à partir de deux types de nucléus, avec chacun leur propre chaîne opératoire : des nucléus "plats" et des nucléus coniques. Ces nucléus coniques, destinés au débitage de petites lames, étaient parfois juste configurés et acheminés tels quels vers les sites consommateurs. Une partie de ces supports était retouchée dans les ateliers en outils unifaciaux, de mor-

(7) D'après les témoins morphologiques de la majorité des produits laminaires (pas ou peu de régularisation du bord de plan de frappe, variation des silhouettes et des proportions, grands talons lisses, points d'impact bien visibles, bulbes esquillés bien prononcés), il est probable que la technique par percussion directe avec de petits percuteurs d'andésite a été employée. Toutefois, nous ne pouvons exclure l'hypothèse d'autres techniques de percussion. phologie répétitive, l'autre partie étant évacuée à l'état brut. Enfin, la préparation de pièces bifaciales constituait également l'une des tâches régulières, quoique secondaire, des artisans. Toutes ces activités se déroulaient conjointement ou alternativement au sein d'un même espace.

Les processus opératoires suivis pour la fabrication des lames faisaient appel à des moyens techniques apparemment simples mais qui requéraient néammoins une excellente habileté. La normalisation globale de ces processus apparaît de façon claire, mettant en valeur le haut degré de spécialisation, la finalité restant la production laminaire. Mais, nous avons remarqué des petites variantes d'ordre technologique, inhérentes à l'amplitude de telles concentrations de débitage et donc forcément aux apports personnels des nombreux artisans qui ont travaillé dans ces ateliers, (à ce propos, nous nous référons aux travaux sur les comportements techniques individuels de S. Ploux, 1989, 1991). Ces variantes révèlent entre autres une capacité d'adaptation et une certaine souplesse dans la manière d'appréhender la matière première et de procéder au débitage. II apparaît clairement que les artisans allaient au plus simple et tiraient parti de la configuration initiale des blocs. La nature de ceux-ci intervenait de manière décisive dans le mode de préparation des nucléus, selon qu'ils présentaient des arêtes naturelles ou une configuration nodulaire. Bien que nous n'ayons pas trouvé de nucléus plats en cours de débitage ou à l'étape de préparation (8), les caractéristiques de ces nucléus à l'état résiduel permet de suggérer un usage prédominant de blocs angulaires.

Le schéma simplifié de la fabrication des lames à partir de ces nucléus plats comporte les étapes suivantes:

1) un dégrossissage partiel et facultatif régularisait le volume exploitable (lorsque cela n'avait pas été fait dans les unités d'extraction, notamment à l'intérieur et à la sortie des mines). Chaque bloc ayant sa propre configuration, les produits détachés étaient de morphologies très diverses, avec, bien entendu, de grandes plages corticales ;

2) la mise en place d'un plan de frappe lisse s'effectuait au moyen d'un enlèvement transversal et l'identification de quelques nucléus à plan de frappe cortical et celle de lames au talon cortical indique la mise à profit très occasionnelle de belles plates-formes naturelles ;

3) le débitage s'effectuait, par percussion, à partir de cet unique plan de frappe et deux types d'approche étaient alors appliqués : les artisans profitaient des arêtes naturelles pour détacher immédiatement des produits laminaires (fig. 5 c), créant ainsi tout de suite des nervures directrices. Ces produits peuvent être en-

(8) Si lors de nos parcours méthodiques, nous n'avons pu recueillir des nucléus plats à ce stade de débitage, rien n'exclut de telles trouvailles lors de prochaines visites, l'ampleur de ces concentrations rendant difficile une couverture exhaustive. 
tièrement corticaux, les produits suivants offrant une facette corticale. Ou bien, lorsque les blocs étaient nodulaires ou qu'ils ne présentaient pas d'arêtes exploitables avec profit, la préparation de la surface de débitage assurait un passage graduel de l'éclat à des produits aux modules laminaires, en aménageant progressivement des nervures, de plus en plus longues et régulières. Dès le début du travail, les artisans s'appliquaient d'ailleurs à extraire des éclats de forme allongée.

Lors du débitage laminaire, les artisans préparaient rarement le bord de plan de frappe. Lorsque cette préparation avait lieu, elle consistait en la supression de la corniche ou en la régularisation par de petits enlèvements. Le débitage se déroulait jusqu'à la réduction maximale du volume disponible. Les nucléus étaient abandonnés lorsque le degré d'aplanissement de leur surface de débitage, et leur faible épaisseur, empêchaient de nouveaux détachements.

Les nucléus qui résultent de ce débitage sont de configuration plate, de forme rectangulaire ou ogivale avec une face débitée opposée à une surface plane, le plus souvent entièrement corticale. Dans quelques cas, cette surface non débitée peut avoir été régularisée par quelques enlèvements. Le plan de frappe, habituellement lisse, est oblique, plus rarement perpendiculaire. Si l'on observe une grande uniformité dans la silhouette finale de ces nucléus, les dimensions, quant à elles, sont très variables et indiquent le détachement de produits aux proportions diversifiées :

- les plus grands nucléus peuvent présenter une hauteur dépassant les $20 \mathrm{~cm}$, avec une largeur variant entre 11 et $17 \mathrm{~cm}$ et une épaisseur comprise entre $4 \mathrm{~cm}$ et $5 \mathrm{~cm}$;

- les nucléus que l'on trouve en proportions majoritaires ont une hauteur moyenne de $15 \mathrm{~cm}$ et une largeur inférieure à $13 \mathrm{~cm}$. L'épaisseur varie entre 3 et $5 \mathrm{~cm}$;

- des petits nucléus de hauteur comprise entre 7 et $10 \mathrm{~cm}$, de largeur comprise entre 5 et $8 \mathrm{~cm}$ et d'épaisseur incluse entre 2 et $4 \mathrm{~cm}$.

Le poids imposant des blocs provenant des mines (pouvant atteindre $25 \mathrm{~kg}$ ) et la présence de grandes lames longues de 25 à $30 \mathrm{~cm}$ donnent une bonne idée des dimensions initiales de certains de ces nucléus. Les produits laminaires détachés lors du débitage sont de morphologies assez diversifiées (fig. 5 d). II apparaît clairement que le produit recherché doit combiner des proportions équilibrées sans pour autant atteindre une perfection des formes qui tendrait à une standardisation des modules.

Pour la préparation des nucléus coniques, le choix se portait sur des blocs de dimension plus réduite, angulaires ou nodulaires et de silhouette oblongue. Des gros éclats provenant du dégrossissage ou de l'aménagement du plan de frappe ont parfois été réutilisés comme support de débitage. Les stades suivis sont grosso modo similaires à ceux adoptés pour le débitage des nucléus plats, avec cependant des particularités propres à la morphologie des supports utilisés et des produits recherchés :

- après une régularisation du volume exploitable, le plan de frappe était obtenu par le détachement transversal d'un grand enlèvement.

A partir de ce nucléus ou bien de l'éclat récupéré, trois possibilités se présentaient alors :

- la mise en forme de la surface de débitage s'effectuait sur le pourtour et assurait la création progressive de nervures directrices de plus en plus longues. II s'agit du procédé le plus fréquent ;

- des arêtes naturelles facilitaient le détachement immédiat de produits laminaires ;

- l'aménagement d'une crête permettait le détachement du premier produit laminaire. Ce mode de préparation semble avoir été adopté occasionnellement et les quelques fragments de lames à crête rencontrés proviennent tous de petits spécimens qui présentent un seul versant retouché, l'autre étant cortical.

La régularisation de la surface du débitage s'effectuait jusqu'à l'obtention de nervures suffisamment régulières permettant le détachement de petites lames (entre $7 \mathrm{~cm}$ et $10 \mathrm{~cm}$ de longueur). Le débitage s'effectuant par percussion, on observe une assez grande variation dans les silhouettes et les proportions, l'équilibre général de la pièce étant le principal critère retenu. Les nucléus étaient soit exploités au maximum de leur capacité puis abandonnés, soit stop- pés au dernier stade de configuration laminaire, auquel cas, nous supposons qu'ils étaient évacués tels quels hors de la zone de production.

Les produits extraits lors du débitage de ces deux types de nucléus avaient un destin variable, qui dépendait de leur position au sein de la chaîne opératoire. Quoique généralement délaissés, les grands éclats d'entame ou de dégrossissage, lorsqu'ils n'étaient pas réemployés à leur tour comme nucléus, pouvaient être réutilisés pour le façonnage des préformes bifaciales foliacées ou amygdaloïdes. Ces dernières pouvaient être aussi préparées sur des produits laminaires larges ou même sur des plaquettes naturelles (elles présentent alors des restes de plage corticale sur les deux faces). Les grandes lames débitées en début de travail des nucléus plats étaient souvent abandonnées, mais quand leurs modules le permettaient, elles servaient de support au façonnage de grands outils unifaciaux. Les grandes lames régulières étaient soit modifiées en outils unifaciaux, soit évacuées à l'état brut. De même, nous supposons que les lames aux dimensions plus réduites étaient acheminées telles quelles, vers les sites consommateurs où elles étaient souvent enmanchées après avoir subi deux encoches bilatérales au niveau de la base ; leur tranchant était utilisé sans modifications autres que les réaffutages nécessaires, effectués au fur et à mesure de l'usure. Enfin, le façonnage des autres produits de préparation apparaît comme exceptionnelle et semble correspondre à des besoins ponctuels en vue d'un usage immédiat.

\section{Les ateliers des Tzinapus del Cerro Prieto}

Ces ateliers offrent en surface des concentrations de matériel dont la nature se distingue très sensiblement de celui des autres ateliers du Cerro Varal et du Cerro Zinaparo. On y observe :

- des éclats de débitage provenant du dégrossissage et des stades de préparation des nucléus. IIs sont nettement majoritaires (en moyenne $60 \%$ du matériel) ; les plus gros déchets sont concentrés dans les talus de déjection ; les modules de ces éclats sont très diversifiés ;

- une forte proportion de produits laminaires (entre $30 \%$ et $40 \%$ 
du matériel) : il s'agit exclusivement de produits courts et assez larges (le rapport de proportion largeur/longueur est compris entre 0.35 et 0.70 ), de dimensions variables. Les produits très allongés sont extrêmement rares ;

- des nucléus coniques configurés pour le débitage de petits produits laminaires correspondant aux normes indiquées ci-dessus (fig. 6 b). Ces nucléus, prêts à l'exploitation, présentent des modules normalisés (dimensions, sithouette et poids) ;

- des "rabots-nucléus" circulaires, au profil trapézoïdal et à base plane (ils ont en fait l'allure de cônes tronqués à leur sommet), aux modules également standardisés (fig. 6 a) ;

- des grandes préformes bifaciales amygdaloïdes délaissées au stade d'ébauche (fig. 5 f);

- des grattoirs circulaires également de morphologie très répétitive ;

- la présence très rare d'autres catégories d'outils (sur éclats ou sur supports laminaires), de grande diversité morphologique.

Lors de l'étude du matériel, plusieurs questions relatives à la fonction réelle de ces "rabots-nucléus" sont apparues. En effet, il s'agit de produits que leur morphologie situe entre nucléus et rabot (9). Plusieurs indices nous indiquaient qu'il s'agissait d'un outillage (ils présentent notamment une hauteur axiale qui est presque toujours inférieure au diamètre du plan de frappe). Toutefois, sur les sites d'habitat approvisionnés, nous retrouvons ces supports à l'état résiduel (au même titre que les nucléus coniques), associés à leurs éclats. Plusieurs hypothèses ont alors été proposées (Darras, à paraître), mettant en valeur leur fonction ambivalente, les réaffûtages successifs des parties actives requérant un traitement en tant que nucléus et entraînant logiquement l'épuisement de leur volume.

En tout état de cause, il apparaît clairement que la finalité du débitage consistait en la préparation de nucléus coniques, configurés pour le

(9) Le terme "rabot" fait référence ici à des instruments plus épais que les grattoirs, de silhouette circulaire. Fréquemment employé dans les études lithiques mésoaméricaines ("cepilo") notamment pour designer des gros tils de basalte ou d'andésite, il ne renvoie pas, malgré son qualificatif implicite, à une fonction particulière.

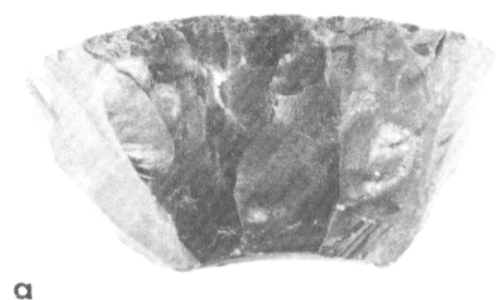

a

exploitable ; des éclats épais et de grandes dimensions (de silhouettes diverses) étaient volontairement extraits en vue de leur réutilisation ;

2a) un plan de frappe lisse était ensuite créé par un grand enlèvement ;

2b) ou bien, une plate-forme naturelle était utilisée comme plan de frappe (plus rare) ;

2c) ou encore, un des gros éclats était réemployé, sa face d'éclatement constituant le plan de frappe ;

3) la mise en forme du nucléus consistait alors à détacher des éclats allongés dès le début du travail, sur le pourtour, puis à créer progressivement des nervures longitudinales permettant le débitage de produits laminaires courts et assez larges, jusqu'à atteindre la configuration requise.

Les nucléus de forme conique qui résultent de ce travail, sont de morphologie très répétitive : plan de frappe circulaire variant entre 9 et $11 \mathrm{~cm}$, hauteurs axiales souvent inférieures et oscillant entre 5 et $11 \mathrm{~cm}$, poids inclu entre 300 et $500 \mathrm{gr}$. Les "rabots-nucléus" offrent des caractéristiques similaires, avec cependant, des hauteurs moins élevées (essentiellement entre 5 et $7 \mathrm{~cm}$ ) et une base plane opposée au plan de frappe et souvent corticale.

Pour la fabrication des grattoirs, des éclats moins épais étaient récupérés dans la masse de déchets produite aux stades du dégrossissage et de mise en forme. La silhouette était alors modelée par percussion. II s'agit de produits standardisés, parfaitement circulaires, dont le diamètre oscille entre 6 et $10 \mathrm{~cm}$ et la hauteur entre 2 et $4 \mathrm{~cm}$.

Les grandes préformes bifaciales recueillies dans les ateliers ont été délaissées au stade d'ébauche. Longues d'une vingtaine de $\mathrm{cm}$, elles présentent une épaisseur marquée et des arêtes sinueuses. La finalité d'une telle production n'a pas été identifiée.

Toutes les opérations de débitage se déroulaient par percussion directe, avec des percuteurs d'andésite de morphologies différentes selon le stade de travail. Pour les dernières étapes, les artisans utilisaient des petits percuteurs discoïdes ou ovoïdes de poids répétitif (entre $300 \mathrm{gr}$ et $400 \mathrm{gr}$ ). 


\section{LES HABITATS}

Outre les nombreux vestiges d'habitats recensés dans l'ensemble du massif de Zinaparo-Churintzio, qui ont fourni des informations sur les modalités d'occupation, quatre centres d'exploitation de l'obsidienne comportent des structures d'habitat en nombre plus ou moins important, localisées généralement en amont des épanchements, dans des zones libres de ce matériau. Ces vestiges sont composés de restes de maisons, installées sur des platesformes aménagées, et parfois associées à des systèmes de terrasses agricoles. Les plates-formes ont été construites avec des remblais de divers matériaux (gros blocs d'andésite, blocs d'obsidienne, terre) puis bordées de parements de dalles équarries. D'un degré d'élaboration très inégal, certaines bases de maisons ont été élevées avec de grandes dalles de ce type et le remblaiement des sols a été réalisé avec des nodules d'obsidienne. Les opérations de sondages ont révélé une absence de stratigraphie (particularité commune à tous les habitats de la région) due à la très faible épaisseur des dépôts (le substratum était atteint entre -20 et $-50 \mathrm{~cm}$ ) et ont donné peu de matériel archéologique, celui-ci se trouvant surtout en surface. II se compose de céramique qui correspond à une vaisselle d'usage domestique, de quelques restes osseux brûlés (cervidés, canidés), de restes de charbons (retrouvés dans l'une des maisons), et d'un matériel lithique qui atteste le caractère domestique des structures (instruments de mouture et industrie taillée). Pour le débitage, leurs occupants utilisaient des petits nodules d'obsidienne au cortex roulé qui semblent provenir de récoltes de surface ainsi que de l'andésite, de provenance locale (du Cerro Grande). Les activités de débitage qui se déroulaient à l'intérieur des maisons et/ou à l'extérieur, sur les plates-formes, correspondaient apparemment à des besoins ponctuels satisfaits dans l'immédiat : les nucléus d'obsidienne sont généralement de forme irrégulière à plusieurs plans de frappe et les éclats d'obsidienne apparaissent le plus souvent utilisés tels quels sans retouches préalables, tandis que les instruments d'andésite sur éclats sont élaborés plus finement. On y retrouve également quelques lames brutes et outils sur lames originaires des ateliers (lames à encoches basales de fixation, grattoirs distaux, couteaux sur lames) ; néanmoins, ces produits ont une faible fréquence par rapport au reste du matériel.

En raison de la contiguité spatiale de ces structures d'habitat avec les zones de travail de l'obsidienne, nous avons admis le postulat de leur contemporanéité et donc d'une étroite corrélation. Toutefois, la nature de leurs relations et leur véritable fonction n'ont pu être déterminées avec certitude. II apparaît clairement que le débitage assuré dans les maisons obéit à des nécessités d'ordre domestique. Aucune correspondance ne peut être établie entre le débitage spécialisé des ateliers qui révèle un haut niveau technologique et ce débitage assez frustre (en ce qui concerne le travail de l'obsidienne). Bien que les besoins aient été indéniablement différents, la faible proportion de produits originaires des ateliers (qu'ils soient des produits recherchés ou bien des produits délaissés ayant pu être récupérés et utilisés) pose le problème du libre accès à la production des ateliers. Plusieurs schémas interprétatifs concernant la fonction de ces habitats peuvent être proposés. II pourrait s'agir de maisons d'artisans et de leurs familles, auquel cas, le débitage intra-muros a pu être réalisé par des personnes non spécialisées dans le débitage de l'obsidienne (femmes, enfants) et ayant un accès limité aux zones de production. La pratique d'activités agricoles, attestée par la présence de terrasses dans l'un des sites et d'instruments de mouture, implique une certaine sédentarité et donc probablement l'occupation permanente des habitats. Toutefois, nous pouvons aussi envisager l'hypothèse d'habitats temporaires abritant ponctuellement des artisans lors de séjours laborieux dans les centres d'exploitation, ou encore de maisons de gardiennage occupées par des individus chargés de contrôler l'accès aux gîtes (10).

(10) A diverses époques de l'histoire préhis panique, les gîtes d'obsidienne des Hauts Plateaux Centraux ont fait l'objet de contrôles (par Teotihuacan par exemple) qui pouvaient limiter leur accès. Cette mainmise, dont on connait mal les mécanismes opératoires, semblait se manifester par un monopole de l'exploitation des gites et par un contrôle des étapes conse desites et par un contróle des étapes consécutives : fabrication et écoulement des produits d'obsidienne.

\section{LA QUESTION CHRONOLOGIQUE}

Dans l'état actuel des connaissances, nous ne disposons d'aucun marqueur direct permettant de dater avec précision la mise en valeur organisée et intensive de ces gîtes d'obsidienne (11). Le caractère très homogène de la production des centres d'exploitation du Cerro Varal et du Cerro Zinaparo suggère une cohésion temporelle et une même appartenance culturelle. Mais, entre le début de la mise à profit méthodique de certaines zones et l'abandon (relatif ou absolu) des derniers centres, un certain temps s'est nécessairement écoulé. D'autre part, au sein d'une même entité de production, il est impossible de déterminer le laps de temps pendant lequel ont été produits les milliers de tonnes de déchets d'obsidienne. Des interruptions de longue durée ont pu se produire sans que nous puissions en rendre compte au regard des amoncellements. De plus, toujours au sein d'un même centre d'activités, rien n'atteste la contemporanéité des vestiges miniers. Les spécificités propres à chaque mode d'extraction peuvent correspondre à des périodes d'exploitation distinctes et être le fait de groupes humains différents. Certaines mines ont pu être creusées bien après l'abandon du mode de production qui incluait une transformation sur place des blocs d'obsidienne, d'autres peuvent avoir été abandonnées puis reprises ultérieurement. Enfin, d'autres formes d'exploitation moins perceptibles et moins systématiques ont dû inévitablement exister, en dehors du cadre chronologique proposé pour dater ces mines-ateliers. Elles seraient le fait probable d'autres populations ayant leurs propres besoins et leurs propres traditions lithiques. Plusieurs questions relatives au mode de fonctionnement se posent alors : mise en exploitation simultanée ou au contraire, mise en valeur successive des zones d'obsidienne au fur et à mesure de leur épuisement et des besoins, rythme et constance des activités de débitage, importance de la main-d'oeuvre spécialisée, modalités d'accès aux gîtes. Les stratégies

(11) Sans grande conviction, nous avons néanmoins tenté des datations grâce à la mé thode d'hydratation des couches superficieles l'obsidienne. Les differents résultats obtenus de l'obsidienne. Les diffèrents résultats obtenus n'ont pu être pris en considération en raison de leur incohérence. 
économiques qui ont régi ces centres d'exploitation ont pu fluctuer dans le temps et influer sur les formes et l'intensité de la production.

Dans un premier temps, en accord avec notre postulat de départ, l'étude des habitats associés aux exploitations et de ceux concentrés dans les autres secteurs du massif de Zinaparo-Churintzio a fourni les principales informations concernant la chronologie, malgré la pauvreté certaine des données archéologiques; les périodes d'occupation ont été déterminées par l'analyse céramique et quelques datations chronométriques. Ensuite, les résultats préliminaires concernant la distribution de l'obsidienne dans les sites d'habitat de la région du Versant Lerma et de Zacapu (étude des séries lithiques, compositions géochimiques d'échantillons pour en déterminer l'origine) nous ont permis de compléter les grandes lignes d'un cadre chronologique que les prochaines recherches s'appliqueront à préciser.

Dès le $4^{\theta}$ millénaire $\mathrm{BP}$, les trois gîtes de la région de Zinaparo servaient de lieu d'approvisionnement aux populations qui vivaient dans la région (Faugère, 1990). Le mode d'acquisition consistait alors en la simple récolte en surface de petits nodules roulés. A partir de ces supports de taille réduite, était pratiqué un débitage d'éclats de morphologies diversifiées.

Les données disponibles pour les époques postérieures concernent le Préclassique final (phase Loma Alta : 0-450 ap. J.-C.) et le Classique (phases Jaracuaro-Lupe : 450-800 ap. J.-C.). Elles indiquent que les gîtes du Cerro Varal et du Cerro Zinaparo fournissaient la région de $\mathrm{Za}$ capu (Darras, 1993) et du Versant Lerma mais dans des proportions modérées, d'autres sources plus ou moins éloignées assurant également l'approvisionnement (Zinapecuaro au Michoacan, Pachuca dans l'état d'Hidalgo et dans une moindre mesure, Penjamo au Guanajuato) (12). A ces époques, l'obsidienne du Cerro Prieto était très peu utilisée malgré sa proximité géo-

(12) L'industrie d'obsidienne cotoyait une industrie taillée sur basalte et andésite. Ces dernières roches étaient utilisées pour la fabrication d'outils spécifiques destinés à des tâches plus lourdes, surement en rapport avec les actigros tranchets, etc. graphique (moins de $10 \mathrm{~km}$ pour certains sites d'habitats). Dans le massif de Zinaparo, aucune trace d'occupation datant du Préclassique et du Classique n'a été identifiée. De même, aucune correspondance n'a pu être établie entre la production des ateliers et la nature des obsidiennes rencontrées dans les sites approvisionnés (il s'agit d'industries à éclats, l'industrie laminaire consistant essentiellement en des lames prismatiques obtenues par pression, en obsidienne verte translucide originaire de Pachuca). Bien que cette absence d'analogie ne soit pas un critère décisif, les caractéristiques de ces séries d'obsidiennes indiquent plutôt une exploitation de petits nodules roulés qui étaient probablement en partie dégrossis sur le gisement. Les visites sur les gîtes devaient donc être sporadiques et consistaient en l'acquisition de nodules de surface ou provenant de petites excavations superficielles.

C'est à la fin du Classique et au début du Postclassique (phases La Joya-Palacio : 800-1200 ap. J.-C.) que nous rattachons les formes d'exploitation systématique et spécialisée, combinant activités d'extraction et activités de débitage, qui supposent un degré d'organisation socioéconomique complexe. En effet, la grande majorité des établissements humains du massif de Zinaparo a été datée de cette période (grâce à la séquence céramique et aux datations chronométriques). Par exemple, l'un des habitats inclus dans la zone d'exploitation de Las Navajas a fourni une date ${ }^{14} \mathrm{C}$ de $839 \pm 57$ ap. J.-C. (date non calibrée). Cette invasion soudaine du massif de Zinaparo-Churintzio coïncide avec la multiplication de hameaux et d'établissements plus importants, observée pour la même époque dans les régions voisines du Versant Lerma (Faugère, 1990) et du Rio Angulo. Les analyses géochimiques ont permis de reconnaître les gîtes de la région de Zinaparo comme principale source d'approvisionnement. C'est aussi à cette époque que nous pouvons établir le lien entre la production à la source et le matériel consommé dans les sites d'habitat régionaux. On y retrouve, en effet, des nucléus résiduels configurés dans les ateliers du Cerro Prieto et leurs déchets associés, ainsi que des outils façonnés sur lames produites à Zinaparo. On y trouve également une industrie à éclats réalisée sur des nodules originaires des mêmes gîtes.
Au Postclassique récent (phase Milpillas : $\mathrm{XIII}^{\mathrm{e}} \mathrm{-XV|^{ \textrm {e } }}$ siècle), nous assistons à une diminution très sensible de l'occupation de ces différentes régions et aucun site de cette époque n'a été identifié dans le voisinage des gîtes. Ce phénomène peut s'expliquer à la fois par la politique centraliste du pouvoir tarasque qui favorise un regroupement des populations autour des lacs du Michoacan (lacs de Patzcuaro, Cuitzeo, Zirahuen) et dans les montagnes avoisinantes, ainsi que par l'instabilité politique de ces régions, situées à la périphérie du territoire contrôlé par les Tarasques. En effet, c'est à cette époque que certains groupes de chasseurs-cueilleurs (regroupés sous l'appellation Chichimèques) qui occupent les contrées désertiques du nord du Mexique, semblent multiplier leurs incursions et fragiliser cette zone frontalière (Kirchhoff, 1943), d'ailleurs protégée par des postes militaires (Faugère, 1990, 1991 ; Zepeda Garcia, 1988). Toujours est-il que les gîtes de Zinaparo-Prieto, localisés dans cette zone limitrophe, semblent avoir été partiellement délaissés au profit d'autres sources d'approvisionnement, telles celles de Zinapecuaro et de Ucareo. Les premiers résultats des caractérisations géochimiques effectuées sur des obsidiennes provenant de sites d'habitat de la région de Zacapu (Joron et alii,1991) et de celle de Patzcuaro (Perlstein Pollard et Vogel, 1991) indiquent en effet une nette préférence pour ces derniers gîtes.

Toutefois, les enjeux économiques ont probablement incité les Tarasques à poursuivre l'exploitation de ces sources, moyennant une modification des stratégies d'acquisition. Si l'absence sur place d'activités de transformation nous paraît de plus en plus sûre, certaines mines ont dû continué à fonctionner de façon épisodique, les blocs étant acheminés tels quels ou dégrossis, hors des zones d'exploitation. C'est le cas notamment de la mine de La Guanumeña qui pourrait avoir été creusée tardivement par les Tarasques. En effet, ces derniers avaient une prédilection pour les obsidiennes, assez rares, de couleur rouge-brun, qu'ils utilisaient pour fabriquer leurs couteaux de sacrifice et d'autres pièces bifaciales (jusqu'à maintenant, nous n'avons jamais observé de débitage laminaire par pression avec cette obsidienne). Dans leur capitale, Tzintzuntzan, des aires 
d'activités lithiques étaient consacrées, entre autres, au travail de ce type d'obsidienne (Perlstein Pollard et Vogel, 1991). De la même façon, nous avons identifié plusieurs sites d'habitat postclassiques, tous localisés à proximité des uns des autres, à une vingtaine de kilomètres au sud des gîtes, qui incluent de petits ateliers spécialisés dans le débitage par pression de lames prismatiques grises. Cela renforce l'hypothèse d'un approvisionnement en matière première brute (13) et de son acheminement vers des sites intermédiaires, situés dans des zones bien contrôlées et chargés de la fabrication de produits spécifiques puis de leur distribution. Ces particularités technologiques contrastent d'ailleurs avec les traditions culturelles précédentes où la production laminaire était réalisée par percussion, les quelques lames obtenues par pression et recueillies dans les sites d'habitat de la fin du classique étant de toute évidence importées des Hautes Terres Centrales.

En tout état de cause, cet abandon partiel n'excluait pas des récupérations sporadiques de produits manufacturés puis délaissés à des époques antérieures dans les ateliers des trois gîtes de la région de Zinaparo ; non plus que la pratique sur place de quelques activités de débitage (dans la mine de La Guanumeña par exemple) ou encore, la récolte en surface de nodules roulés.

\section{CONCLUSION}

A une époque, où en Préhistoire, le développement des études technologiques permet d'accéder à une connaissance extrêmement précise des comportements techniques et sociaux, on ne peut que reconnaître les ressources potentielles que recèlent de tels complexes archéologiques et la richesse d'informations qu'ils pourraient encore livrer. Toutefois, l'approche de ces gigantesques laboratoires se heurte aux inévitables problèmes méthodologiques qui limitent la portée de certaines préoccupations scientifiques. En réalité, on peut s'interroger sur la rentabilité de l'investissement temporel et humain qu'exigerait une étude exhaustive et qui relèverait d'une entreprise titanesque. Ceci dit, cette première ap-

(13) L'examen préliminaire des collections d'obsidienne recueillies dans ces sites indiquent une production à partir de blocs probablement dégrossis dans les gîtes. proche a permis l'identification des principales formes de production dans les mines-ateliers d'obsidienne de Zinaparo-Prieto et ouvre de riches perspectives, notamment en ce qui concerne les mécanismes de distribution dans les régions de l'Occident du Mexique.

\section{Bibliographie}

DARRAS V. (1987) - Nota informativa : primeros resultados de la caractérización química por medio de los elementostraza de los yacimientos de obsidiana en la región de Zináparo-Purepero Michoacán, Mexico. Trace 12 CEMCA, Mexico, p. 76-79.

DARRAS V. (1989) - Las Estacas: un grupo original de grabados rupestres en Michoacán, Mexico. Trace 16, CEMCA, Mexico, p. 100-112.

DARRAS V. (1991) - Technologies préhispaniques de l'obsidienne: les centres d'exploitation de Zinaparo-Prieto (Michoacan, Mexique). Thèse de Doctorat de l'Université de Paris-I.

DARRAS V. (1993) - La lítica tallada de los complejos Loma Alta y Lupe. Arqueología de las Lomas en la cuenca de Zacapu, Michoacán. Coord: Arnauld M.C., Carot P., Fauvet-Berthelot M.F., Cuaderno Estudios Michoacanos, $n^{\circ} 5$, chap. 6, CEMCA, Mexico.

DARRAS V. et RODRIGUez F. (1988) - Identificación y explotación de los materiales líticos regionales en el sector de Zináparo, Michoacán. Primera Reunion sobre les Sociedades Prehispanicas en el Centro Occidente de Mexico. Memoria. INAH, Queretaro, Cuaderno de trabajo, $n^{\circ} 1$, p. 139-147.

DarRas V. et Monnet J. (1990) - Une carte rupestre précolombienne ? La modélisation des pétroglyphes mexicains. Mappemonde 90/2, p. 36-40, Reclus, Montpelliers.

DEMANT A. (1981) - L'axe néovolcanique transmexicain. Étude volcanologique et pétrographie, signification géodynamique. Thèse d'État, Université d'AixMarseille.

Demant A. (1983) - Les gisements d'obsidienne de l'axe transmexicain. Bulletin du CEMCA, n ${ }^{\circ}$ 5, p. 23-37, Mexico.

DEMANT A. (1989) - Résultats de la caractérisation géochimique des éléments en trace des gites de la région de Zinaparo, Michoacan. Document de travail, archives du projet Michoacan, CEMCA, Mexico.

ERICSON J.E. et KIMBERLIN J. (1977) - Obsidian Sources, Chemical Characterization and Hydratation Rates in West Mexico. Archaeometry 19 (2), p. 157 166, Great Britain.

FAUGere F. (1990) - Entre nomades et sédentaires: la zone sud du Versant Lerma, Michoacan, Mexique. Thèse de Doctorat de l'Université de Paris-l.

FAugere F. (1991) - San Antonio Carupo (Centro-Norte de Michoacán. Mexico) : Nuevas evidencias de ciertas transformaciones en el inicio del Postclásico. Journal de la Société des Américanistes LXXVII, Paris, p.45-61.
Joron J.-L., Demant A., Darras V. (1990) - Détermination de l'origine d'obsidiennes archéologiques du Michoacan nord-occidental (Mexique), par la géochimie des éléments en traces. Compte-rendu de l'Académie des Sciences, t. 311, Série II, p. 1513-1520.

KIRCHHOFF P. (1943) - Los recolectorescazadores del norte de México. El Norte de México y el Sur de Estados Unidos. Illa Mesa Redonda, Mexico, SMA, p. 133-143.

Michelet D., Arnaulo M.C., Fauvet-BerTHELOT M.F. (1989) - El proyecto del CEMCA en Michoacán. Etapa : un balance. Trace 16, p. 70-87, CEMCA, Mexico.

Pastrana A. (1987) - El proceso de trabajo de la obsdiana de las minas de Pico de Orizaba. Boletín de Antropología Americana 13, p. 133-146, Instituto Panaméricano de Geografía e Historia, México.

PLoux S. (1989) - Approche archéologique de la variabilité des comportements techniques individuels. L'exemple de quelques tailleurs magdaléniens à Pincevent. Doctorat de l'Université de Paris-X-Nanterre.

PLoux S. (1991) - Technologie, technicité, techniciens : méthode de détermination d'auteurs et comportements techniques individuels. 25 ans d'études technologiques en Préhistoire : Bilans et perspectives. Actes des rencontres 18-20 oct.1990, CRAVCNRS, Antibes, 201-214.

Pollard Perlstein H. (1982) - Ecological Variation and Economic Exchange in the Tarascan State. American Ethnologist 9 (2), p. 250-268.

Pollard Perlstein H. et Vogel A. (1991) - Late Postclassic Imperial Expansion and Economic Exchange within the Tarascan Domain. Communication préparée pour le $47^{\text {th }}$ International Congress of Americanists (New Orleans, july 7-11), symposium "Aztec Archaeology : Trade, Production and Economic Issues".

Stocker T.L. et Cobean R.H. (1984) Preliminary report on the Obsidian Mines at Pico de Orizaba, Veracruz. Prehistoric Quarries and Lithic Production, ed J.E Ericson et B.A Purdy, Cambridge University Press, p. 83-97.

TIXIER J., ROCHE H., INIZAN M.L. (1980) Préhistoire de la pierre taillée : terminologie et technologie. CREP/CNRS, Valbonne.

ZePEVADA GarcIa G. (1988) - Nogales : fortaleza tarasca en el Estado de Guanajuato. Primera Reunión sobre las Sociedades Prehispánicas en el Centro-Occidente de México.Memoria. Centro regional de Queretaro, Cuaderno de trabajo 1, INAH, Mexico, p. 299-307.

Véronique DARRAS C.N.R.S. UMR 9959/GRAL Université Toulouse Le Mirail 31000 Toulouse

Dessins des pièces lithiques de Françoise Bagot (CEMCA, Mexico). 